\title{
The effects of surgery on gastrointestinal motor activity
}

E.M.M. Quigley ${ }^{1}$ and J.S. Thompson ${ }^{2}$

\author{
${ }^{1}$ Department of Internal Medicine and ${ }^{2}$ Department of Surgery, \\ Section of Gastroenterology and Hepatology, \\ University of Nebraska Medical Center, Omaha, NE, USA
}

\section{Correspondence \\ E.M.M. Quigley \\ Internal Medicine \\ Gastroenterology and Hepatology \\ University of Nebraska \\ Medical Center \\ 600 S. 42nd St. \\ Omaha, NE 68198-2000 \\ USA \\ Fax: (402) 559-9004 \\ Presented in part at the \\ XII Annual Meeting of the \\ Federação de Sociedades de \\ Biologia Experimental, Caxambu, \\ MG, Brasil, August 27-30, 1997. \\ Research supported in part through the Merit Review Program of the Veterans' Administration.}

Received January 6, 1998 Accepted March 18, 1998

\begin{abstract}
Gastrointestinal surgical procedures have the potential to disrupt motor activity in various organs of the gastrointestinal tract or, indeed, throughout the entire alimentary canal. Several of these motor effects have important clinical consequences and have also served to advance our understanding of the regulation of gastrointestinal motor activity. This review will focus, in particular, on the effects of surgery on the small intestine, and will attempt to emphasize the implications of these studies for our understanding of small intestinal motility, in general.
\end{abstract}

Key words

- Motility

- Motor activity

- Small intestine

- Surgery

- Intestinal resection

- Intestinal transplantation

.....................

\section{Gastrointestinal motor activity: an overview}

The gastrointestinal motor apparatus includes intestinal smooth muscle, the enteric nervous system, autonomic nerves and ganglia, and the central nervous system (Figure 1). Therefore, contractile activity, generated by the intestinal smooth muscle, may be regulated at any one of a number of levels. The generation of motor activity relies, in the first instance, on the basic electrophysiological properties of intestinal smooth muscle. Thus, intracellular electrophysiological recordings from intestinal smooth muscle have revealed an omnipresent slow wave which represents the continual depolarization/repolarization of the membrane potential of intestinal smooth muscle cells. Under basal conditions, this depolarization does not reach the critical level for firing and generation of an action potential - slow waves are not, therefore, associated with contractile activity. Further depolarization beyond the level achieved by the slow wave, triggered by either electrical or chemical stimuli, leads to the generation of the action potential and smooth muscle contraction. In this way, slow waves regulate the phasic contractile frequency for any particular site in the gastrointestinal tract. A second intrinsic property of smooth muscle cells, fundamental for the generation of coordinated motor activity, is the presence of areas of electrical closecontact between cells - nexuses. By virtue of these low resistance areas, electrical signals can be transmitted between smooth muscle cells leading them to act as a functional syncytium. In this manner, impulses can be transmitted both longitudinally and circumferentially over considerable areas of the intestine without needing to invoke any neural transmission. In the same way, areas of greater excitability or spontaneous activity can serve as pacemakers - the electrical signal which originates in these areas can be transmitted to and will entrain adjacent smooth muscle cells. It is now thought that 
Figure 1 - A schematic presentation of the gut illustrating the components of the motor apparatus that may be disrupted by surgical procedures. These include the intestinal muscle layers (circular and longitudinal), the enteric nervous system (submucous and myenteric plexuses) and extrinsic autonomic nerves.

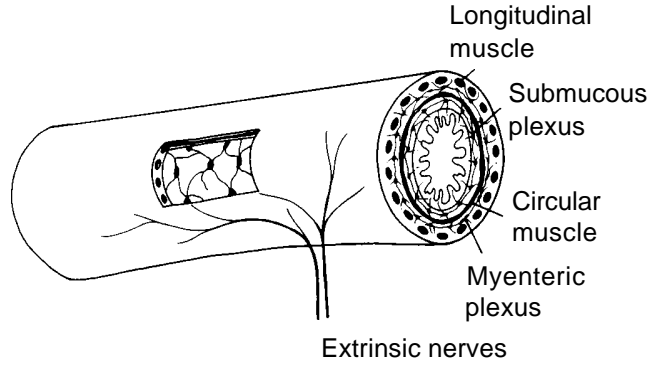

the slow wave originates in specialized muscle cells located in the plexus muscularis profundus and closely related to the deeper layers of the circular muscle layer - the interstitial cells of Cajal - and is then transmitted to the smooth muscle layers. Recent research has also served to emphasize the complexity and importance of the enteric nervous system, which appears capable of generating complex neuronal responses and, thereby, highly organized motor patterns, entirely independent of the autonomic or central nervous systems.

Recordings of intestinal motor activity from a number of species, including man, have demonstrated that motility is organized into two basic patterns - fasting and postprandial $(1,2)$. During fasting, motor activity is organized into a recurring cycle of events: the migrating motor complex (MMC) (Figure 2A-C). Each migrating motor complex comprises three cycles which occur in sequence and continue to recur as long as the individual remains fasted. In man, each cycle lasts 90-120 min and begins with a period of motor quiescence, phase 1 . This is followed by irregular phasic contractile activity, phase 2 , and culminates in a burst of uninterrupted rhythmic, phasic contractions, phase 3 . Phase 3 is the most distinctive component of the cycle and is often utilized as a surrogate for the entire cycle (Figure 2A,B). Phase 3 is thought to originate in the proximal duodenum and migrate slowly in an aboral fashion along the small intestine to the distal ileum (Figure 2C). While in the dog, a commonly used model of in vivo motor activity, phase 3 complexes can be identified at the ileocecal sphincter and even in the proximal colon (Figure 3), MMC activity tends to peter out in the distal ileum in man. Phase 3 of the migrating motor complex is also associated with intense phasic contractile activity in the gastric antrum (Figure 2), and tonic contraction of the proximal stomach, lower esophageal sphincter and gall bladder. Secretory components have also been identified and include increases in gastric acid and pepsin output as well as increased pancreatic and biliary flow in association with duodenal phase 3. Following meal ingestion, MMC activity is abolished and is replaced by intense, apparently irregular contractile activity throughout the stomach and small intestine (Figure 4). This apparently random activity could well be visualized as subserving the important mixing functions associated with meal ingestion. The duration of this fed pattern is variable and depends on the size and caloric content of the meal, lasting anywhere from 2 to $5 \mathrm{~h}$ after a typical meal.

Throughout this review reference will be made to the effects of various surgical procedures on these principal motor phenomena: the slow wave, the MMC and the fed pattern. Studies of the regulation of these motor patterns have revealed how various levels of control are exerted in the regulation of motor activity. It is now evident, for example, that the migrating motor complex is generated and propagated entirely within the gut wall by the enteric nervous system. It can be influenced by autonomic and central input phase 2, in particular, appears to be dependent on central input, and disappears in man during sleep. The fed response, in contrast, is dependent on the integrity of autonomic innervation and the vagus, in particular. Hormonal factors also play a role. Thus, motilin released from endocrine cells in the duodenal mucosa during the intense contractile activity of phase 3 leads to the generation of intense contractile activity in the antrum and together with a vagal mechanism, or- 
chestrates the antral, gastric, lower esophageal, gall bladder and secretory components of phase 3 .

\section{The effects of surgery on motility: an overview}

Surgical procedures may exert several effects on motor activity. The most common manifestation is postoperative ileus, which can be regarded as almost a physiological response to intra-abdominal surgery (3). The duration and severity of postoperative ileus appear to be directly proportional to the extent of the surgical procedure and to manipulation of the intestines, in particular. The pathophysiology of postoperative ileus has been the subject of extensive study details of this important topic are beyond the scope of this review. Our focus, instead, is on the potential effects of extrinsic denervation, transection and reanastomosis, resection, interposition and transplantation on intestinal motor activity (4).

\section{Effects of transection and reanasto- mosis on small intestinal motor activity}

While an incomplete transection of the

Figure 2 - The migrating motor complex (MMC).

$A$, Recording of intraluminal pressure activity from the proximal small intestine in man. Note the various components of the migrating motor complex which include phase 2, irregular phasic contractions (seen on the left of this figure), phase 3 , a band of rhythmic phasic contractions that slowly migrates through the gut (seen in the center of the figure), and phase 1 , motor quiescence (on the right hand side of this figure). This cycle comprising phases 1-3 will continue to occur as long as the individual remains fasted.

$B$, Migrating motor complex. Recording of myoelectrical activity from a dog. Note phase 3 , represented by an uninterrupted band of spike activity, migrating through the proximal small intestine.

$C$, Schematic presentation of an entire 4-h-fasting recording from a dog. Each dot denotes phase 3 of the migrating motor complex. Note that phase 3 recurs at a regular interval of approximately $90 \mathrm{~min}$ and migrates through the small intestine.
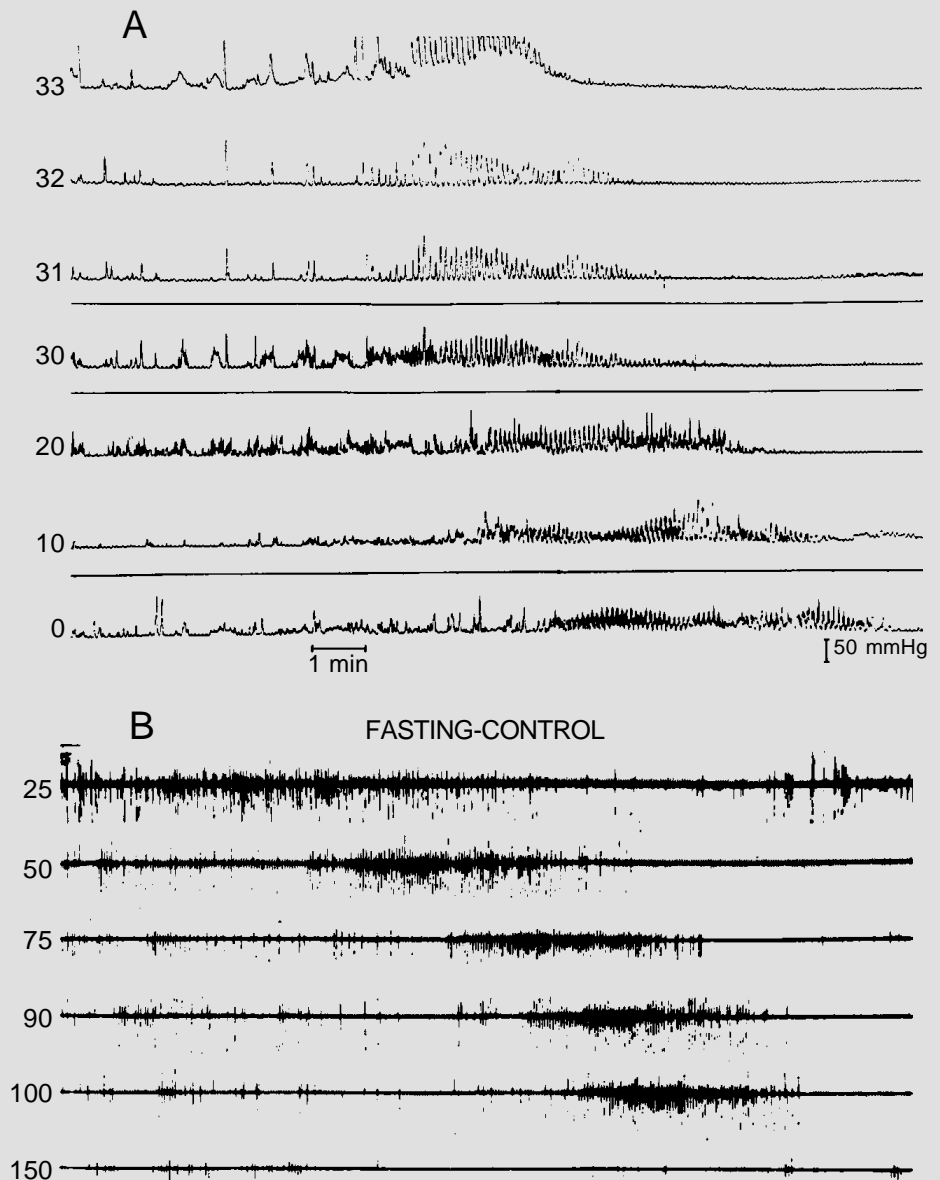

200
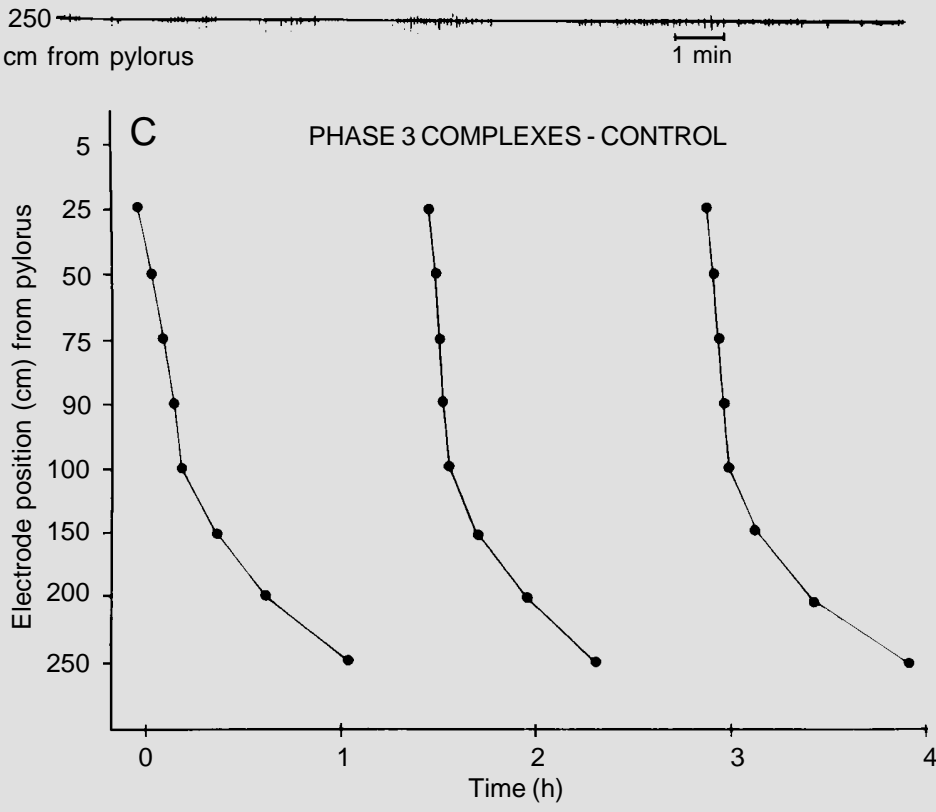
CANINE ILEO-COLONIC JUNCTION - MMC PASSING INTO COLON

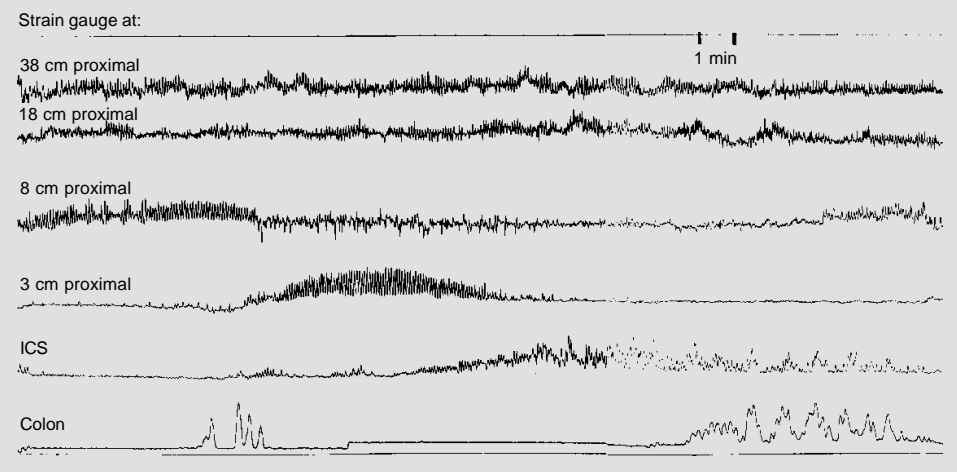

Figure 3 - Fasting motor activity of canine ileo-colonic junction. Note phase 3 of the migrating motor complex migrating through the distal ileum, across the ileocecal sphincter (ICS) into the colon.

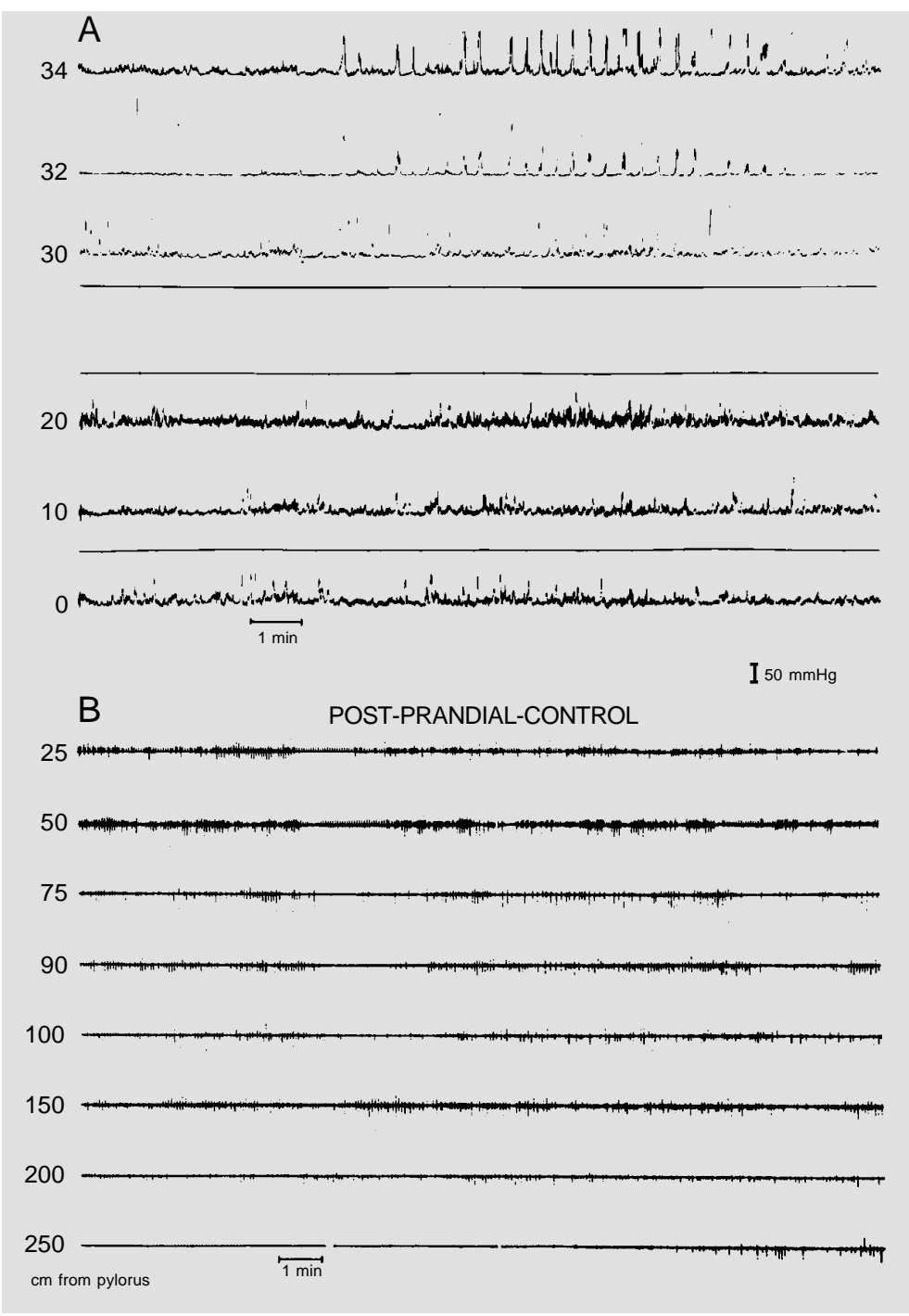

bowel wall does not appear to be associated with any effects on the transmission of myoelectrical signals along the small intestine, a complete transection has clear effects on motor function (5-7). Following a conventional transection and reanastomosis, the transmission of slow wave activity along the small intestine is interrupted $(4,7)$. Loss of myogenic continuity (Figure 5A) means that the intestine distal to the site of transection is no longer entrained by the normally dominant pacemaker located in the proximal duodenum, and now operates at its own intrinsic slow wave frequency (Figure 5B). As a consequence, a sharp drop in slow wave frequency will be observed across the anastomosis. These observations have, indeed, led to the concept of longitudinal, myogenic transmission of slow wave activity. Accordingly, meticulous approximation of the muscle layers at the time of surgery has been shown to preserve myogenic transmission and a normal slow wave gradient (8). Observations on the propagation of migrating motor complex activity across a transection and reanastomosis have produced variable results. In general, there appears to be some evidence of motor asynchrony across the site of transection, though long-term studies suggest reasonable apparent coordination of the MMC across the transection. In interpreting such data, one needs to exert caution and to resist the temptation to describe complexes on either side of an anastomosis as synchronized, when in fact they are merely coincident. If propagation does indeed "recover" across anastomoses, does this reflect regeneration of enteric nerves or a role for extrinsic nerves in the coordination of motor activity (9)? This issue remains unresolved. Re-

Figure 4 - The postprandial motor response. On feeding, migrating motor complex activity is lost and, as illustrated in recordings of intraluminal pressure activity (A) and myoelectrical activity (B) from the proximal small intestine in man and dog, respectively, is replaced by intense irregular activity. 
flecting the primacy of extrinsic innervation in its regulation, transection and reanastomosis have little effect on the fed response $(7,9)$. From a clinical point of view, these various effects of transection and reanastomosis on motor activity appear to have little impact on intestinal homeostasis and have not been associated with any significant effects on digestion or absorption.

\section{Extrinsic denervation}

The model of autotransplantation has provided considerable insights into the role of extrinsic innervation in the regulation of intestinal motor activity. It has also served to provide insights into the potential effects of intestinal transplantation on motor activity. In our own studies in the dog, autotransplantation involved the complete removal of the entire jejunoileum and its subsequent reimplantation into the animal (9). All nerves, blood vessels and lymphatics were, therefore, severed (Figure 6A,B). As expected, and given the presence of an anastomosis at the proximal and distal ends of the transplanted segment, slow wave frequency fell within the transplanted intestine, and indeed, when followed for up to 18 months after the surgical procedure showed no evidence of recovery to normal levels.

Studies of migrating motor complex activity revealed several interesting points. In the immediate aftermath of autotransplantation, migrating motor complexes were severely disrupted, perhaps reflecting ischemic or reperfusion injury to the enteric nervous system. With time, however, MMC activity recovered within the transplanted segment, and by 18 months normal appearing phase 3 complexes could be identified in all animals and were shown to propagate in an orderly fashion through the transplanted segment (Figure 6C). Coordination with the proximal intact intestine did not, however, recover and abnormal patterns continued to be evident within the transplanted segment. These

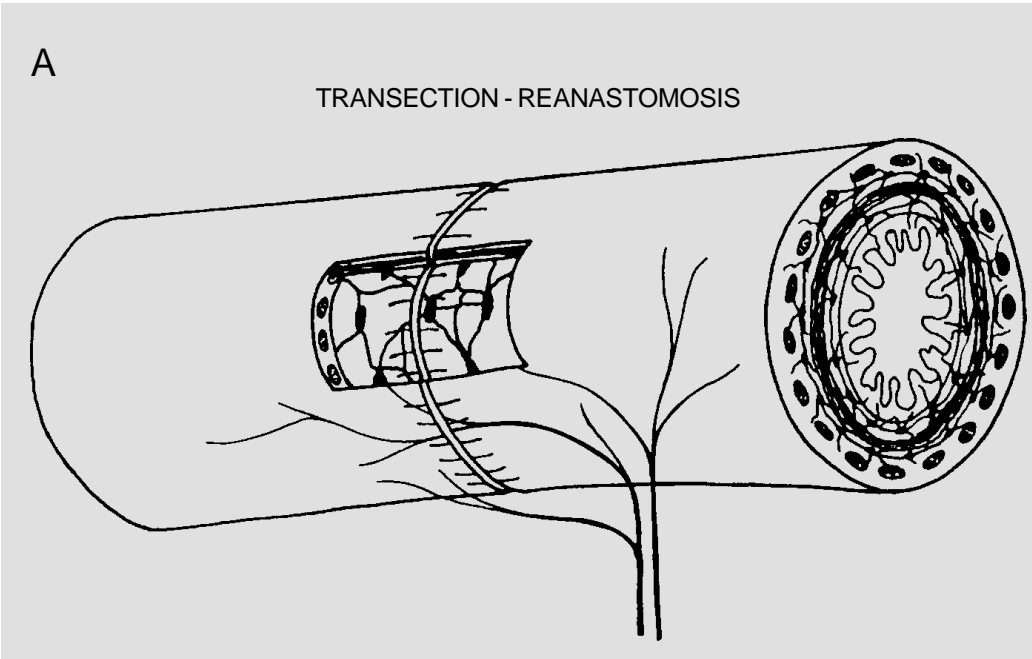

Loss of enteric neural continuity

B

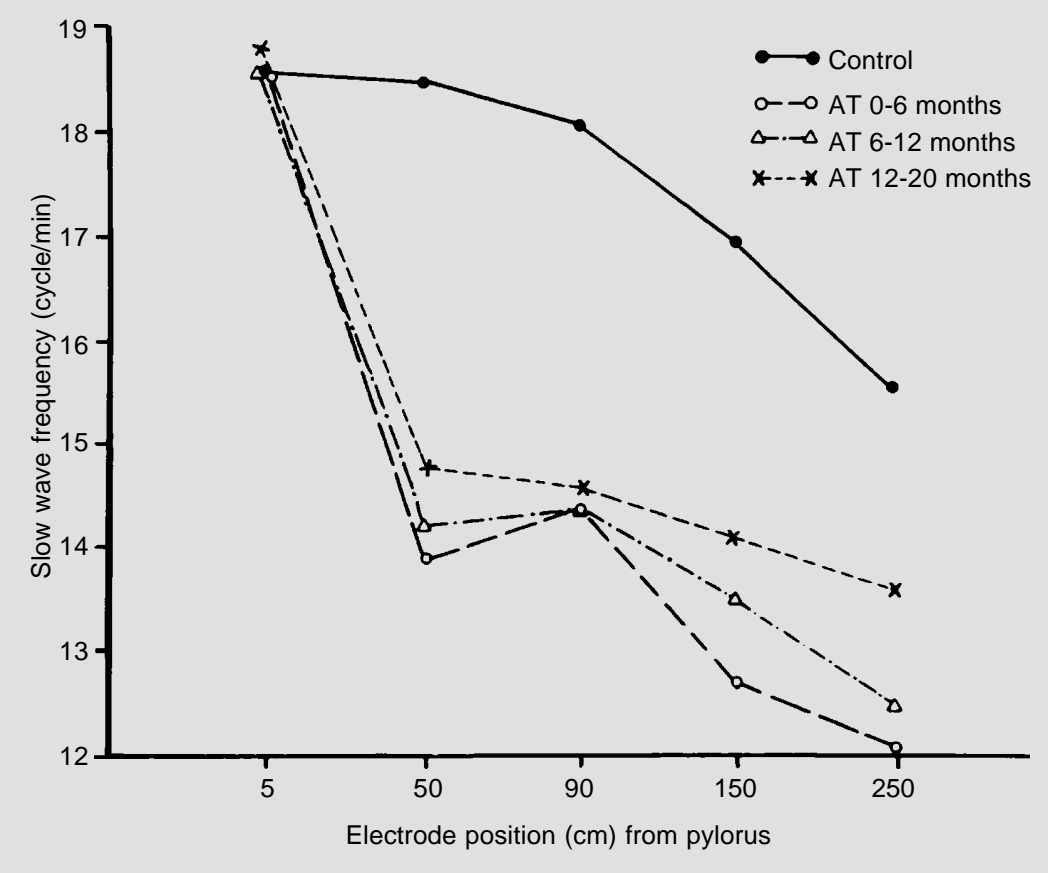

Figure 5 - Effects of intestinal transection on intestinal motor activity.

$A$, Schematic presentation illustrating the effects of transection on intestinal motor apparatus. A complete transection disrupts the longitudinal continuity of intestinal muscle and the enteric nervous system.

$B$, Effects of transection on slow-wave frequency in the canine small intestine. Graphic representation of slow wave frequency along the canine small intestine in control animals and in three groups of animals who had undergone jejunoileal autotransplantation (AT) and were studied at varying intervals following the procedure. Electrode at $5 \mathrm{~cm}$ was located in the intact duodenum. All other electrodes were distal to the site of transection and reanastamosis in autotransplanted bowel. Note sharp drop at slow wave frequency distal to site of transection and reanastamosis in all groups. 


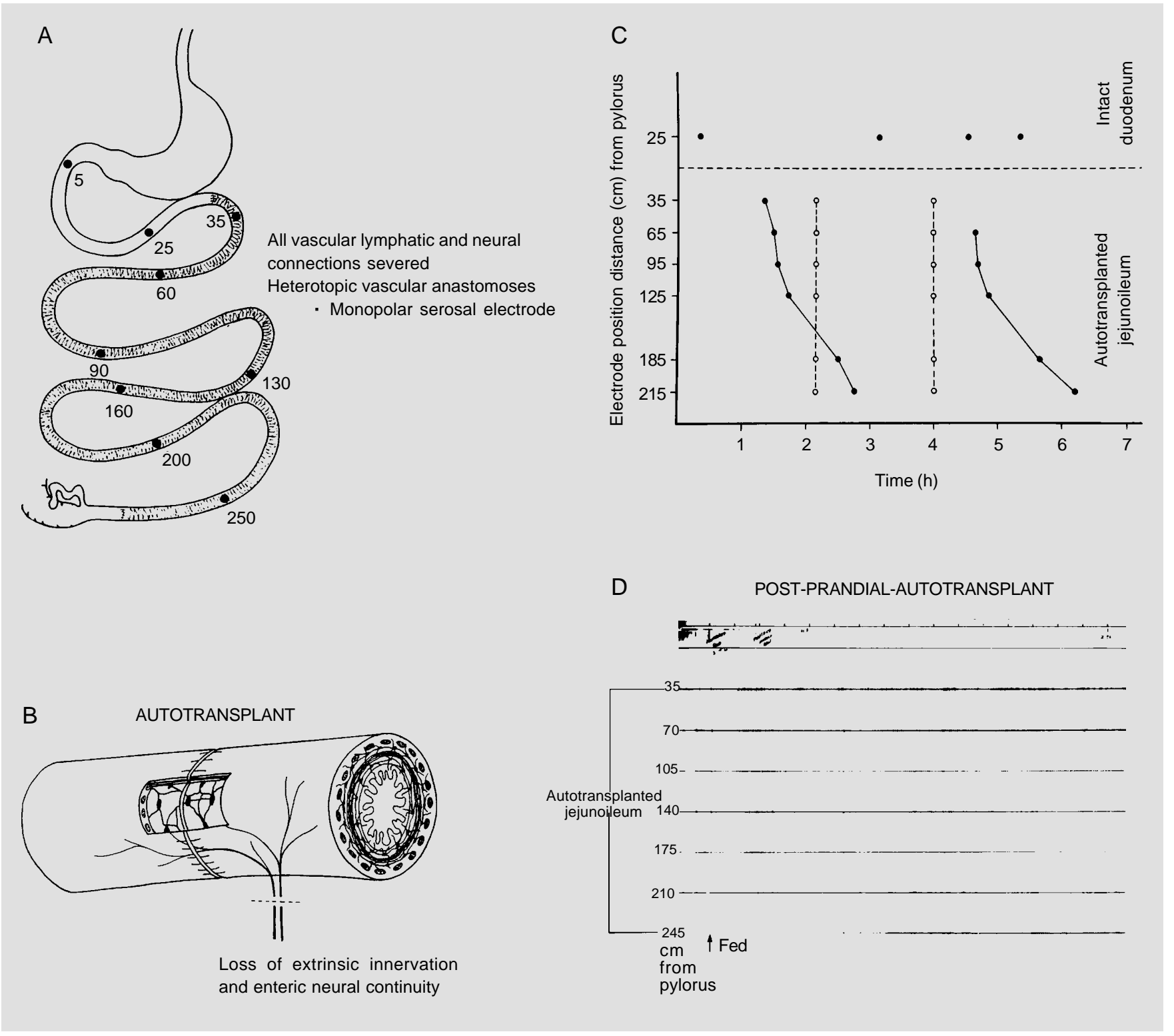

Figure 6 - Effects of autotransplantation (total extrinsic denervation) on intestinal motor activity in a canine model.

A, Autotransplant model. Note that autotransplantation involved removal and reimplantation of the entire jejunoileum with heterotopic vascular anastamoses and complete disruption of all nerves and lymphatics to the autotransplanted segment.

$B$, Schematic presentation of the effects of autotransplantation on the intestinal motor apparatus. Autotransplantation involves disruption of longitudinal continuity of intestinal muscle and the enteric nervous system, as well as complete extrinsic denervation.

$C$, Effects of autotransplants on migrating motor complex. In this part of an entire fasting recording from an autotransplanted dog, note retention and normal propagation of phase 3 of the migrating motor complex (denoted by filled circles) through the autotransplanted segment. Phase 3 also recognized in intact proximal duodenum but there is a complete lack of coordination between phase 3 activity in the intact duodenum and the autotransplanted segment. Open circles represent propagating phasic bursts.

$D$, Effects of autotransplantation on postprandial motor response. Note the complete absence of postprandial motor response in the autotransplanted segment. 
observations are perhaps the most dramatic demonstration of the ability of the enteric nervous system to independently generate and propagate the migrating motor complex $(10,11)$. Differences were observed in the coordination of the migrating motor complex across the transection between transplanted animals and those who had a transection and reanastomosis alone, suggesting a role for extrinsic nerves in the coordination of MMC activity along the length of the intestine. Another important observation in these studies was the complete and permanent abolition of the typical fed motor response, confirming the important role of extrinsic nerves in the mediation of this motor pattern (9-11).

In these studies, recordings from control animals suggested that the response to a meal included an immediate, brief, rapidly migrating motor event in the proximal intestine, followed by the typical fed pattern which migrated more slowly and persisted. In the auto-transplanted animals, the typical fed response was consistently abolished; a delayed and relatively transient pattern of fedtype motility was observed, however, perhaps reflecting a luminal phase of the fed response (Figure 6D). Morphological studies confirmed the integrity of the enteric nervous system within the transplanted segment (12) and studies of circulating and tissue peptide levels, though demonstrating significant changes following transplantation, did not reveal any significant correlation between these changes and the observed motor events (13).

Similar, though less dramatic, effects have been documented following either truncal vagotomy or sympathetic denervation (4). Vagotomy leads to loss of the accommodation reflex and will, therefore, tend to accelerate liquid emptying; impaired antral contractility, in contrast, will lead to delayed gastric emptying of solids. When combined with an emptying procedure such as a pyloroplasty or gastroenterostomy, however, the net effect is little change in the rate of emptying of a mixed meal $(2,4)$. Vagotomy has also been associated with a shortening of the interval between MMC cycles and with an impaired fed response, phenomena which may contribute to some of the observed clinical effects of vagotomy on transit and absorption (4).

\section{The intestinal motor response to resection}

While the biochemical, immunological, hormonal and morphological responses of the small intestine to extensive resection have received considerable attention (14), little is known of the motor response to removal of extensive parts of the small intestine. To address this issue, we have recently completed a series of studies, in the dog, on the motor response to resection. In the first of these studies, we compared motor activity in the intestinal remnant following resection of 25,50 , and $75 \%$ of the distal intestine (15). In the short-term, resection was associated with dramatic changes in motor activity in the remnant, these changes being most evident in animals that had undergone a $75 \%$ resection. In the distal remnant, in the 25 and $50 \%$ resection groups, and throughout the remnant in the $75 \%$ resection animals, motor activity was dominated by intense clustered

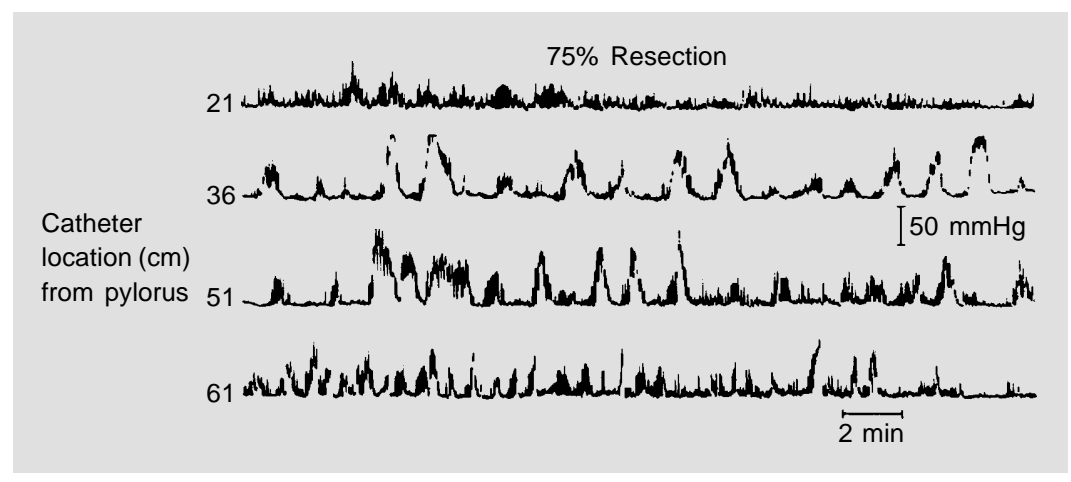

Figure 7 - The motor response to an extensive intestinal resection. Recordings of motor activity from the intestinal remnant in an animal following resection of the distal $75 \%$ small intestine. Note the dominance of intense "cluster" activity throughout the intestinal remnant. 
contractions (Figure 7). These clusters (defined as periods of rhythmic phasic activity separate from phase 3 of the migrating motor complex) appeared disordered, and, though sometimes propagated in an aboral direction, were often simultaneous or even retrograde (16). Clustered activity has been demonstrated in a number of pathological conditions in man and has been associated with such symptoms as abdominal cramping and pain. This clustered activity is also reminiscent of the contractile pattern which normally dominates motor activity in the most distal small intestine in both dog and man $(6,17,18)$, and raises the possibility that its presence in the more proximal intestine following resection could represent exposure to a luminal environment normally associated with the distal ileum. While cluster activity dominated following resection, phase 3 of the migrating motor complex was preserved. Indeed, in a subsequent comparison of the effects of distal and proximal resection, there was no significant difference in the prevalence of MMC phase 3 complexes in the remaining intestine in either group (19). There was, however, some evidence for disruption of aboral propagation following distal resection. Of further interest, the proximal resection was not associated with the development of any of the abnormal patterns associated with distal resection.

Given the intensity of this motor response following distal rather than proximal resection and its relationship to the extent of the resection, it was tempting to speculate that it might be mediated by altered luminal contents. Given the prior demonstration that distal ileal-type motor patterns such as clusters and prolonged propagating contractions (giant migrating contractions) could be induced by the installation of volatile fatty acids into the intestinal lumen (20), bacteria and their products were especially attractive candidates. We did not, however, observe any relationship between bacteria or volatile fatty acid content and the motor response in the above-mentioned comparison between distal and proximal resection. To evaluate this further and also to examine the possible role of the ileocecal sphincter, we compared the motor response to distal resection among animals with an intact ileocecal sphincter and in those in which the ileocecal sphincter was bypassed by direct ileocolonic anastomosis (21). While the latter group demonstrated, as expected, more steatorrhea and increased intraluminal concentrations of bacteria and volatile fatty acids in the distal ileum, we could not demonstrate any difference in the motor response to resection between the two groups. These observations suggested firstly that, in contrast to the mucosal response (22), the motor response to resection was not mediated by the presence of a colonic-type flora in the distal remnant $(23,24)$, nor by exposure to increased concentrations of volatile fatty acids (a product of bacterial metabolism of undigested carbohydrate), and, secondly, that the presence or absence of the ileocecal sphincter did not influence the motor response to resection. The impact of loss of the ileocecal sphincter on the digestive and absorptive function of the intestinal remnant was again confirmed.

Our studies to date, therefore, do not support a role for bacteria or their products in the generation of this motor response. This does not exclude an effect of other bacterial products such as conjugated bile acids, for example. It is also possible, with reference to the ileocecal bypass studies, that loss of the distal ileum rather than the ileocecal sphincter may be more relevant to the motor changes. Thus, all of our distal resection studies involved loss of the most distal ileum, a region which has important and unique absorptive and motor functions. Loss of the receptor site for bile salt absorption, for example, could lead to bile salt deficiency and to motor changes. There is some evidence to suggest a role for bile salts in maintaining the migrating motor complex, for example (25). Similarly, loss of the unique 
motor properties of the distal ileum could precipitate motor dysfunction in the intestinal remnant $(26,27)$. Other factors could also be relevant. Extensive resection of the distal small intestine is associated with significant changes in circulating peptides of enteric origin, and, in particular, with increased basal and postprandial levels of the peptides gastrin, CCK, glucose-dependent insulinotropic peptide (GIP), peptide YY (PYY), and enteroglucagon (28). None of these changes appear, however, to correlate with the motor events. The relationship to PYY is deserving of further study, however. PYY appears to mediate the ileal brake - a homeostatic reflex whereby the instillation of fat into the terminal ileum retards gastric emptying and small intestinal transit (29). Studies of tissue levels of peptides revealed reduced levels of vasoactive intestinal peptide (VIP) and increased levels of calcitonin gene-related peptide (cGRP) in mucosa and muscle (28). The reduced levels of VIP, an important inhibitory peptide, might well explain the prominence of motor disruption following resection.

We have also studied the possibility that these motor responses could reflect a fundamental change in the morphology or physiology of the intestinal muscle layer (30-32). In a separate evaluation of the morphology of the various layers of the intestinal musculature (32), as well as in in vitro studies of length-tension relationships and the response to a cholinergic agonist (31), we have failed to identify any abnormalities in these parameters of remnant smooth muscle structure and function.

To date, therefore, these studies have demonstrated that extensive resection of the distal small intestine is associated with significant disruption in the proximal remnant this disruption does not, however, appear to be mediated by changes in intraluminal bacterial or volatile fatty acid content nor is it related to changes in circulating or enteric peptides. Some preliminary evidence sug- gests that this motor response may adapt (33). Thus, when studied over three months, transit seems to slow in the distal remnant and cluster activity tends to diminish, suggesting that adaptation may occur in a manner analogous to that seen in the mucosa (33). The time scale of this adaptation appears, however, to be different, with motor adaptation occurring over months, rather than days or weeks. Studies in man also suggest motor adaptation; recordings performed years following resection do not reveal active cluster activity, but rather a shortening of the MMC period and of the relative durations of phases 1 and $2(34,35)$. The postprandial motor response was also significantly shorter following extensive distal resections.

\section{Motor effects of restorative procedures}

Over the years, several surgical procedures have been advocated to improve di-

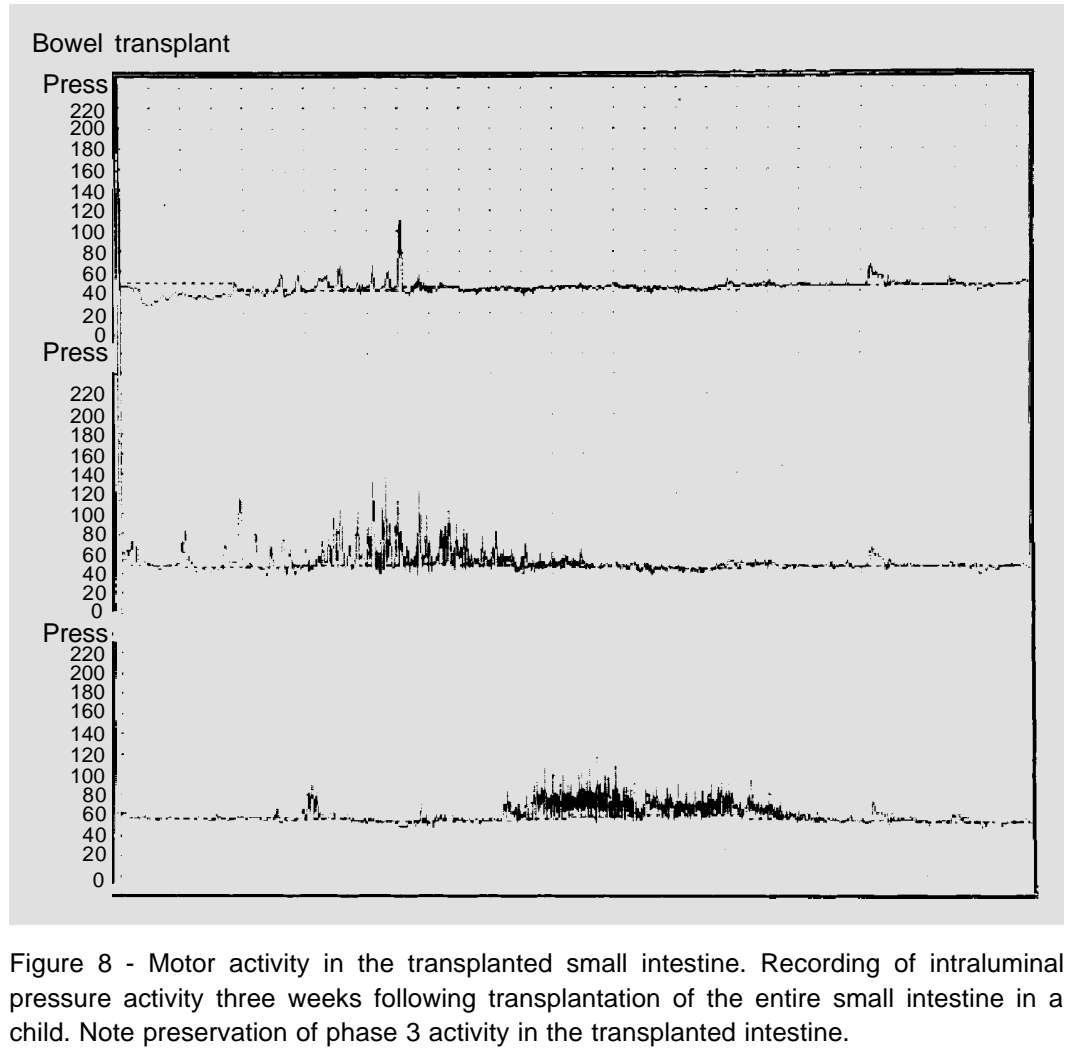


gestive and absorptive function following extensive resection of the distal small intestine $(36,37)$. Some of these procedures, such as the creation of artificial valves or sphincters and the interposition of colonic or reversed intestinal segments, have attempted to retard transit through the remnant, whereas others, such as serosal patching and transplantation, sought to increase mucosal surface area.
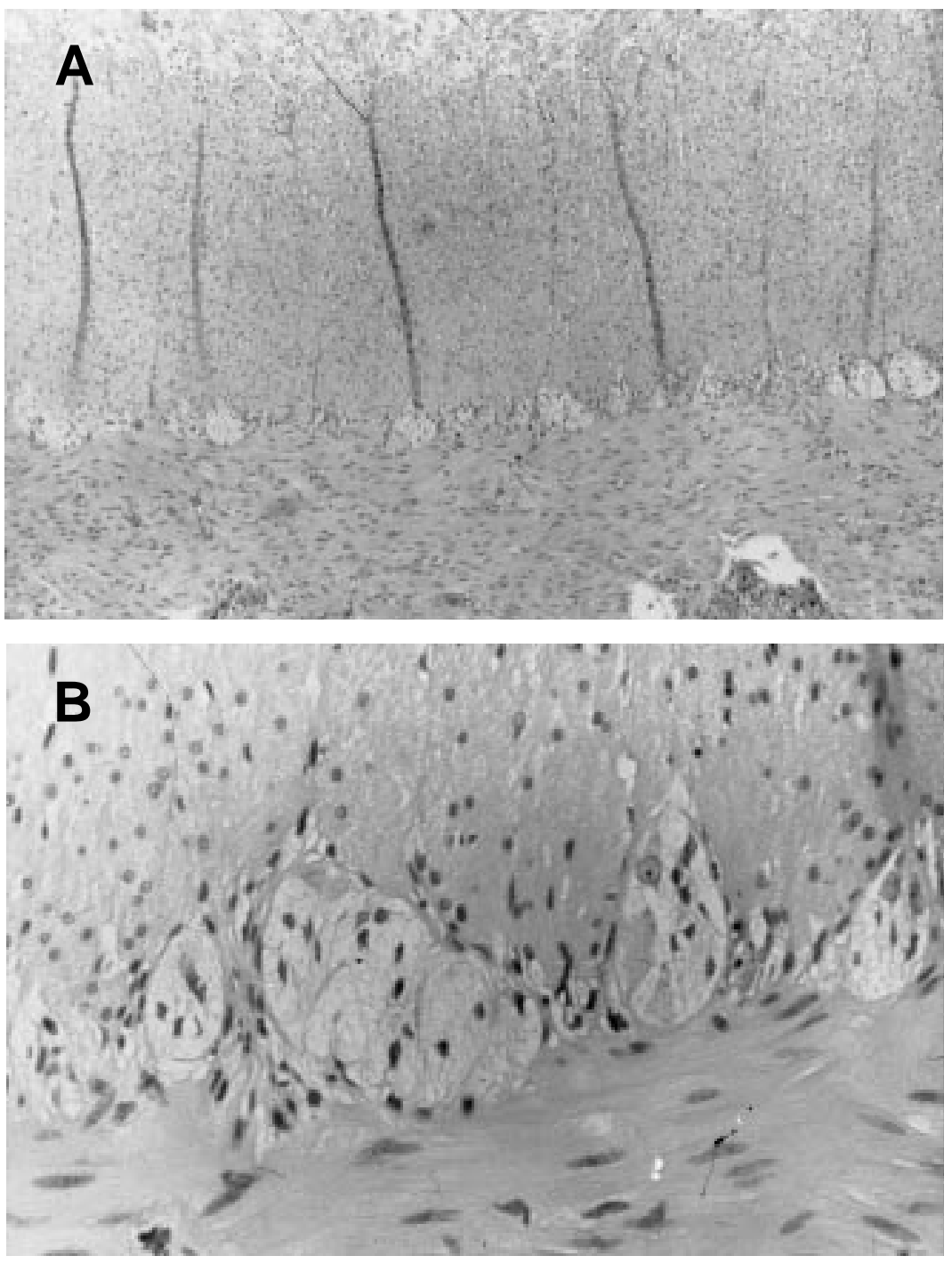

Figure 9 - Morphology of the intestinal motor apparatus in the transplanted intestine. $A$, Low-power magnification (hematoxylin and eosin stain) view of a cross-section through the small intestinal wall obtained at the time of restoration of intestinal continuity, six months following successful small intestinal transplantation. Note intact, normal-appearing, muscle layers (circular above, longitudinal below). $B$, High-power view of the myenteric plexus from the same specimen. Neuronal cell bodies in the myenteric plexus appear dilated and vacuolated.
In the intact intestine, placement of an artificial sphincter in the form of an inverted nipple valve promotes proximal cluster activity (7). A detailed evaluation of the effect of a similar sphincter in animals that have undergone an extensive distal resection failed, however, to demonstrate any influence of the sphincter on the frequency or characteristics of motor patterns in the proximal remnant (38). The reported beneficial clinical effects of a sphincter substitute do not appear, therefore, to be related to a modification of the motor response to resection but may simply reflect those of a low-grade mechanical obstruction.

The interposition of a reversed jejunal segment was associated with motor asynchrony and with a reversal of propagated motor events within the reversed segment, but had relatively minor effects on motor patterns in the proximal remnant (39). One interesting observation was the occasional recording of rapidly propagated, retrograde events, reminiscent of those associated with vomiting, in the intestine proximal to the reversed segment.

Serosal patching, a procedure whereby a defect is created in the small intestine and then "patched" onto the colonic serosa, leads to the ingrowth of neomucosa to fill the defect, but does not result in any motor disruption in the small intestine, nor is it associated with the propagation of small intestinal myoelectrical patterns into the colon (40).

Intestinal tapering and lengthening attempts to increase absorptive surface area. A segment of the intestinal remnant is divided along its longitudinal axis and the divided segments are joined by an end-to-end anastomosis, thereby, in theory at least, effectively doubling the length of the remnant. This procedure was also not associated with any major disruption or modulation of motor patterns in the remnant $(41,42)$.

Taken together, these findings suggest that restorative procedures have relatively 
minor effects on the motor response to resection and that their proposed beneficial influence on the adaptive response to resection is not mediated through an effect on motility.

Relatively few studies have examined the motor consequences of heterotopic, allogeneic transplantation of the small intestine $(9,43,44)$. In an animal model $(44)$, as well as in limited observations in man (45), intact migrating motor complex activity has been identified in the transplanted intestine (Figure 8), in accordance with the above-described observations in the auto-transplanted animal model. In a rat model of chronic rejection, Bauer and his group (46) have observed that, in contrast to acute rejection, which primarily involves the intestinal mucosa, chronic rejection appeared to be associated with significant immune-mediated injury to intestinal nerves and muscles. This observation has not, as yet, been corroborated in man, though we have been concerned by the identification of vacuolated enteric neurons in full thickness specimens obtained at the time of reconstitution of intestinal continuity in some of our patients (Figure 9). If the observations in the animal model hold true, chronic rejection could lead to the eventual development of a chronic pseudo-obstruction syndrome.

\section{Conclusions}

The effects of various surgical procedures on intestinal motor activity have been reviewed. While subtle changes in myoelectrical or motor activity can be identified following many of these procedures, their clinical impact appears, for the most part, minimal. Extensive resection is, however, associated with a significant disruption of motor activity in the proximal remnant, and could well explain some of the symptoms reported by these patients. The mediation of this effect remains unclear, but it does not appear to reflect changes in intraluminal bacterial or volatile fatty acid content. Although the fed motor response is lost, several complex motor patterns are preserved following total transplantation of the small intestine the integrity of the enteric nervous system $(47,48)$ with its ability to generate and propagate many important motor patterns may explain the relative normality of motor function following such an extensive procedure.

\section{References}

1. Quigley EMM (1987). Small intestinal motor activity - its role in gut homeostasis in health and disease. Quarterly Journal of Medicine, 65: 799-810.

2. Quigley EMM (1996). Gastric and small bowel motility in health and disease. Gastroenterology Clinics of North America, 25: 113-145.

3. Bollinger S \& Quigley EMM (1994). Disordered gastrointestinal motility. In: Quigley EMM \& Sorrell MF (Editors), Medical Care of the Gastrointestinal Surgical Patient. Williams \& Wilkins, Baltimore, 157-174.

4. Quigley EMM (1994). The effect of resection, restorative procedures and transplantation on intestinal motility. In: Kumar D \& Wingate DL (Editors), An Illustrated Guide to Gastrointestinal Motility. 2nd edn. Churchill Livingston Co., Edinburgh, 691716 .
5. Quigley EMM, Phillips SF, Dent J \& Taylor BM (1983). Myoelectrical activity and intraluminal pressure of the canine ileocecal sphincter. Gastroenterology, 85: 10541062.

6. Quigley EMM, Phillips SF \& Dent J (1984). Distinctive patterns of interdigestive motility at the canine ileocolonic junction. Gastroenterology, 87: 836-844.

7. Quigley EMM, Thompson JS \& Lof J (1989). Disruption of canine jejunal interdigestive myoelectrical activity by an artificial ileocecal sphincter. Studies of the intestinal motor response to a surgicallyfashioned sphincter substitute. Digestive Diseases and Sciences, 34: 1434-1442.

8. Galligan JJ, Furness JG \& Costa M (1989). Migration of the myoelectric complex after interruption of the myenteric plexus: intestinal transection and regeneration of enteric nerves in the guinea pig. Gastroenterology, 97: 1135-1146.

9. Quigley EMM, Spanta AD, Rose SG, Lof J \& Thompson JS (1990). Long-term effects of jejunoileal autotransplantation on myoelectrical activity in the canine small intestine. Digestive Diseases and Sciences, 35: 1505-1517.

10. Sarna S, Condon RE \& Cowles V (1983). Enteric mechanisms of initiation of migrating myoelectric complexes in dogs. Gastroenterology, 84: 816-822.

11. Matsumoto T, Sarna SK, Condon RE, Cowles VE \& Franitzides C (1980). Differential sensitivities of morphine and motilin to initiate migrating motor complex in isolated intestinal segments. Regeneration of intrinsic nerves. Gastroenterology, 90: 61-67.

12. Quigley EMM, Adrian TE, Christensen J, 
Herrington MK, Johnson TJ, Lof J, Rose SG \& Thompson JS (1990). Normal enteric peptides and neuronal morphology in the autotransplanted gut. Gastroenterology, 96: 1228 (Abstract).

13. Adrian TE, Quigley EMM, Rose SG, Johnson TJ \& Thompson JS (1994). Effects of jejunoileal autotransplantation on gastrointestinal regulatory peptides. Digestive Diseases and Sciences, 39: 24572466.

14. Dowling RH \& Booth CC (1967). Structural and functional changes following small intestinal resection in the rat. Clinical Science, 32: 139-149.

15. Quigley EMM \& Thompson JS (1993). The motor response to intestinal resection: motor activity in the canine small intestine following distal resection. Gastroenterology, 105: 791-798.

16. Quigley EMM \& Thompson JS (1994). Clustered motor activity - the dominant motor pattern in the short bowel syndrome. An analysis of regional and temporal distribution. Transplantation Proceedings, 26: 1451-1452.

17. Quigley EMM, Borody TJ, Phillips SF, Wienbeck M, Tucker RL \& Haddad A (1984). Motility of the terminal ileum and ileocecal sphincter in healthy man. Gastroenterology, 87: 857-866.

18. Quigley EMM, Phillips SF, Cranley B, TayIor BM \& Dent J (1985). Tonic pressures at the canine ileocolonic junction: topography and relationship to phasic motor activity. American Journal of Physiology, 249 (Gastrointestinal and Liver Physiolo$g y, 12):$ G-350-G-357.

19. Quigley EMM, Thompson JS, Adrian TE \& Lof $J$ (1996). The motor response to intestinal resection is site specific. Gastroenterology, 110: 740 (Abstract).

20. Quigley EMM, Lof J, Johnson TJ, Jacobs DL \& Mandel KG (1990). Modification by Mebeverine of volatile fatty acid-induced terminal ileal motor patterns. Gastroenterology, 96: 1227 (Abstract).

21. Thompson JS, Quigley EMM \& Adrian TE (1998). Role of the ileocecal junction in the motor response to intestinal resection. Journal of Gastrointestinal Surgery, 2: 174-185.

22. Thompson JS, Quigley EMM, Palmer JM, West WW \& Adrian TE (1996). Luminal short chain fatty acids and postresection intestinal adaptation. Journal of Parenteral and Enteral Nutrition, 20: 338-343.

23. Thompson JS \& Quigley EMM (1997). Intestinal flora and nutrient absorption after intestinal resection. Journal of Gastrointestinal Surgery, 1: 554-560.

24. Thompson JS, Quigley EMM \& Adrian TE
(1998). Qualitative changes in enteric flora and short chain fatty acids after intestinal resection. Digestive Diseases and Sciences, 43: 624-631.

25. Hellstrom PM, Nilsson I \& Svenberg $T$ (1995). Role of bile in regulation of gut motility. Journal of Internal Medicine, 237: 395-402.

26. Phillips SF, Quigley EMM, Kumar D \& Kamath PS (1988). Motility of the ileocoIonic junction. Gut, 29: 390-406.

27. Quigley EMM (1988). Motor activity of the distal ileum and ileocecal sphincter and its relation to the region's function. Digestive Diseases, 6: 229-241.

28. Adrian TE, Thompson JS \& Quigley EMM (1996). Time course of adaptive regulatory peptide changes following massive small bowel resection in the dog. Digestive Diseases and Sciences, 41: 11941203.

29. Nighingale JMD, Kamm MA, Van Der Sijp JRM, Ghatei MA, Bloom SR \& LennardJones JE (1996). Gastrointestinal hormones in short bowel syndrome. Peptide YY may be the "colonic brake" to gastric emptying. Gut, 39: 267-272.

30. Nguyen BL, Thompson JS \& Quigley EMM (1995). Regional variation in canine intestinal muscle mass and function. Digestive Diseases and Sciences, 40: 14911497.

31. Thompson JS, Quigley EMM, Lassiter D \& Adrian TE (1996). Smooth muscle contractility after intestinal resection. Journal of Surgical Research, 60: 379-384.

32. Nguyen BL, Thompson JS \& Quigley EMM (1996). Effect of extent of resection on intestinal muscle adaptation. Journal of Surgical Research, 61: 147-151.

33. Quigley EMM, Thompson JS \& Adrian TE (1995). Motor adaptation following extensive resection of the small intestine. Gastroenterology, 108: 673 (Abstract).

34. Remington $M$, Malagelada JR, Zinsmeister A \& Fleming CR (1983). Abnormalities in gastrointestinal motor activity in patients with short bowels: effect of a synthetic opiate. Gastroenterology, 85: 629-636.

35. Schmidt $\mathrm{T}$, Pfeiffer $\mathrm{A}$, Hackelsberger $\mathrm{N}$, Widmer R, Meisel C \& Kaess H (1996). Effect of intestinal resection on human small bowel motility. Gut, 38: 859-863.

36. Thompson JS, Langnas AN, Pinch LW, Kaufman S, Quigley EMM \& Vanderhoof JA (1995). Surgical approach to shortbowel syndrome. Annals of Surgery, 222: 600-607.

37. Thompson JS (1995). Surgical aspects of the short-bowel syndrome. American Journal of Surgery, 170: 532-536.
38. Quigley EMM \& Thompson JS (1994). Effects of an artificial ileocolonic sphincter substitute on motility in the intestinal remnant following subtotal small intestinal resection. Digestive Diseases and Sciences, 39: 1222-1229.

39. Thompson JS, Quigley EMM \& Adrian TE (1995). Effect of reversed intestinal segments on intestinal structure and function. Journal of Surgical Research, 58: 1927.

40. Thompson JS \& Quigley EMM (1991). Effects of small intestinal serosal patch on small intestinal and colonic motor activity: The effects of a lateral enterotomy on small intestinal myoelectrical activity. Journal of Investigative Surgery, 4: 203215.

41. Thompson JS, Quigley EMM \& Adrian TE (1994). Effect of intestinal tapering and lengthening on intestinal structure and function. American Journal of Surgery, 169: 111-119.

42. Adrian TE, Thompson JS \& Quigley EMM (1996). Enteric neuropeptide abnormalities following small bowel resection and the effect of a lengthening and tapering procedure. Transplantation Proceedings, 28: 2552-2553.

43. Sarr MG \& Hakim NS (1994). Enteric Physiology of the Transplanted Intestine. RG Landes Co., Austin, TX.

44. Pernthaler H, Kreczy A, Plattner R, Pfurtscheller G, Saltwari L, Schmid T, Ofner D, Klima S \& Margreiter R (1994). Myoelectric activity during small bowel allograft rejection. Digestive Diseases and Sciences, 39: 1216-1221.

45. Ikoma A, Nakado K, Suzuki T, Nakamura K, Reynolds JC, Todo S \& StarzI TE (1994). Gastrointestinal motility in the immediate postoperative period after intestinal transplantation, with special reference to acute rejection. Transplantation Proceedings, 26: 1657-1658.

46. Heeckt PF, Lee KK, Halfter WM, Schraut WH \& Bauer AJ (1995). Functional impairment of enteric smooth muscle and nerves caused by chronic intestinal autograft rejection regresses after FK506 rescue. Transplantation, 59: 159-164.

47. Murr MM, Miller VM \& Sarr MG (1996). Contractile properties of enteric smooth muscle after small bowel transplantation in rats. American Journal of Surgery, 171: 212-218.

48. Sarr MG, Siadati MR, Bailey J, Lucas DL, Roddy DR \& Duenes JA (1996). Neural isolation of the jejunoileum. Journal of Surgical Research, 61: 416-424. 